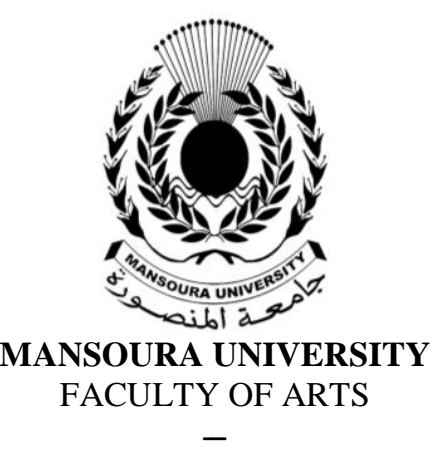

\title{
LA MUSIQUE DE LA BELLE EPOQUE D'APRÈS JEAN CHRISTOPHE DE ROMAIN ROLLAND
}

\author{
Présenté par \\ Dr. Maha Ibrahim Salama
}

Maître de conférences

Faculté de Jeunes Filles -Université Ain shams

Journal of The Faculty of Arts- Mansoura University

$58^{\text {th }}$ ISSUE- Jan. 2016 


\section{LA MUSIQUE DE LA BELLE EPOQUE D'APRÈS}

\section{JEAN CHRISTOPHE DE ROMAIN ROLLAND}

\section{Maha Ibrahim Salama}

A partir du XIX ${ }^{\text {ème }}$ siècle, musique, peinture et littérature suivent les mêmes courants. D'un mouvement Romantique vers une école Réaliste puis un courant Naturaliste pour aboutir au début du $X^{\text {ème }}$ siècle, à la période de la Belle Époque, vers un pseudo Romantisme, à un pseudo Symbolisme et à un pseudo Classicisme. Cependant les mots de Verlaine «De la musique avant toute chose $»^{1}$, de Hugo $« \mathrm{La}$ musique, c'est du bruit qui pense $»^{2}$, voici Théophile Gautier se plaindre en disant que «La musique est le plus désagréable et le plus cher de tous les bruits $»^{3}$, toutes ces définitions sonnent à nos oreilles comme « un écho sonore » et nous invite à étudier la situation de la musique selon la conception de Romain Rolland.

$\mathrm{Si}$ nous consultons une galerie de tableaux de cette période, nous trouvons les instruments de musique qui remplissent les toiles comme dans le tableau de Paul Cézanne, Jeune fille au piano (Fig 1), dans le tableau d'Edgar Degas, L'orchestre de l'Opéra (Fig 2) et dans le tableau d'Edouard Manet, Le fifre (Fig 3). De même, plusieurs romans s'intéressent au côté artistique, peinture et musique, comme nous allons le voir dans Jean Christophe de Romain Rolland qui décrit les tableaux de la peinture avec toutes ses décorations :

«Dans le salon de l'hôtel aristocratique, décoré de tapisseries un peu pâles, avec, sur un chevalet, au milieu de la pièce, le portrait de la robuste Mme Stevens par un peintre à la mode qui l'avait représentée languissante comme une fleur sans eau, les yeux mourants, le corps tordu en spirale, pour exprimer la rareté de son âme millionnaire. ${ }^{4}$

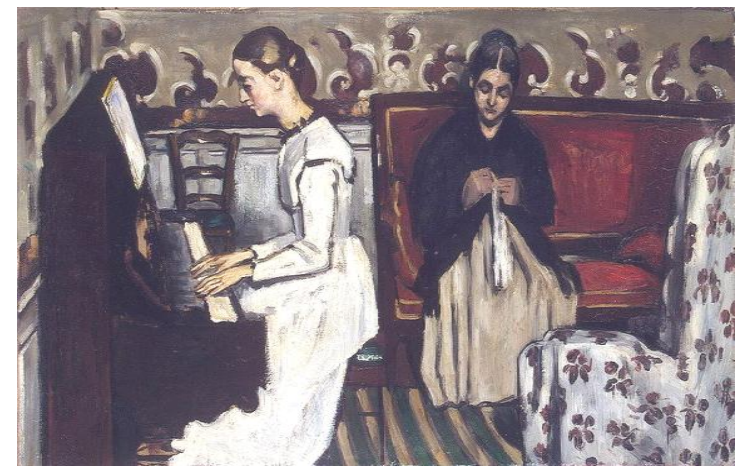

Figure 1, Jeune fille au piano (1869)

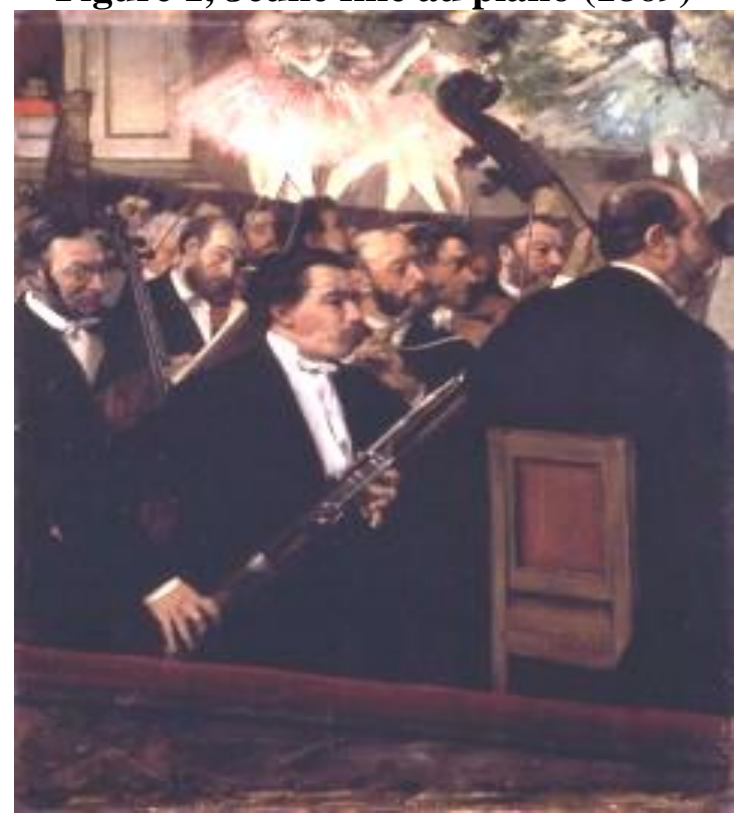

Figure 2, L'orchestre de l'Opéra (1868)

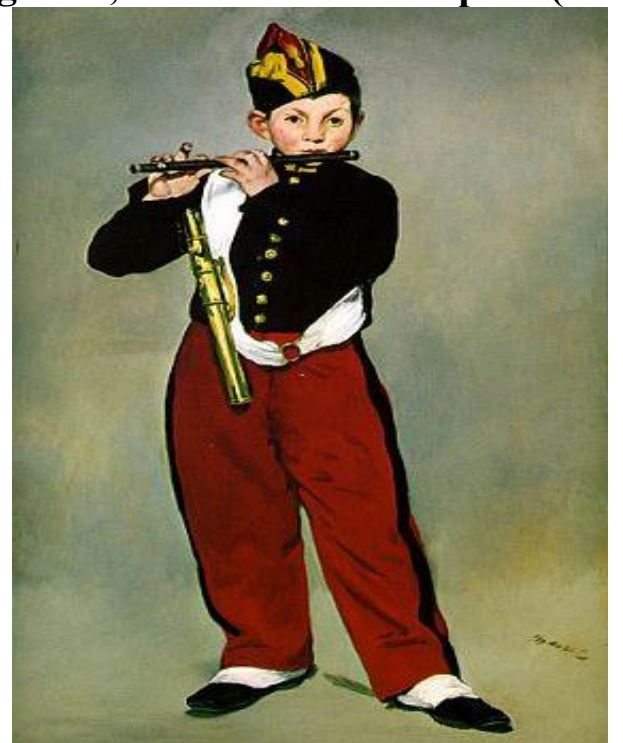

Figure 3, Le fifre (1866) 
C'est aussi l'amour de la musique qui nous a fait découvrir l'écrivain, l'historien et le musicologue français du début du $X X^{\text {ème }}$ siècle, Romain Rolland, qui avoue que :

«La musique m'a tenu par la main, dès mes premiers pas dans la vie. Elle a été mon premier amour, et elle sera probablement le dernier. $\gg^{5}$

Né à Clamecy à la Nièvre en 1866, il perd la foi dès son enfance en lisant les philosophes et, toute sa vie, il cherche une foi nouvelle : tantôt du côté de l'art, tantôt du côté de la révolution. La première de ces conversions se fait, grâce à sa mère, sous le signe de la "divine musique" qui seule, ditil, sait "faire vibrer l'âme". Il s'est très tôt initié aux œuvres des grands maîtres de la musique, Mozart avant tout.

Agrégé d'histoire en 1889, il fut envoyé pour deux ans à l'École d'archéologie de Rome. L'Italie était pour lui une source merveilleuse d'inspiration. Malwida, une amie établie depuis longtemps à Rome, Allemande aux origines françaises, l'initia vers des horizons nouveaux. Ayant connu Mazzini, Wagner, Nietzsche ; grâce à elle, Romain Rolland a pris une conscience plus nette de la pensée et de l'art européens.

Pendant cette période, il réunissait les éléments de sa future thèse de doctorat sur L'Histoire de l'opéra en Europe avant Lulli $^{6}$ et Scarlatti ${ }^{7}$. D'ailleurs une seule figure s'imposait progressivement dans son esprit et son cœur: celle de Jean Christophe :

«J'étais penché sur l'esplanade qui domine les jardins Corsini, la conque de mes oreilles emplie par la musique fastueuse des fontaines, et rêvant... Soleil couchant. A mes pieds flambait la ville, rouge sombre, en hémicycle. Le sourire des monts Albains, à l'horizon, s'alanguissait. Et du Soracte, sur la campagne, 1'Arche flottait (...). $)^{8}$

De retour à Paris, son ambition était de fonder un théâtre populaire analogue à celui du moyen âge et des Grecs. Il y traitera des sujets qui intéresseront les masses, il y présentera des sentiments simples et violents, par réaction contre les spectacles à la mode vers 1900 comme Les Loups (1898), Le Triomphe de la raison (1899), Danton (1900), Le Quatorze Juillet (1902). Il rédigea aussi des articles musicaux sur la musique italienne, sur Mozart, sur Berlioz, sur Siegfried de Wagner. Tout en donnant des cours d'Histoire de l'Art à l'École Normale Supérieure, il organise des cours d'histoire de la musique à l'École des Hautes Études Sociales et il inaugure l'enseignement de cette discipline à la Sorbonne. Romain Rolland a été le Secrétaire général du Congrès d'Histoire de la musique en 1900 créant la Revue d'histoire et de critique musicales, entre 1904 et 1912, il se consacre à la rédaction de Jean Christophe:

«La pensée de Jean Christophe couvre plus de vingt années de ma vie. La première idée date du printemps 1890, à Rome. Les derniers mots écrits sont de juin 1912. L'œuvre déborde au-delà de ces limites. J'ai retrouvé des ébauches de 1888, alors que j'étais encore élève à l'École Normale supérieure de Paris. ${ }^{9}$

Ce roman-cycle, qui paraît d'abord dans la revue de Péguy, Les Cahiers de la Quinzaine, s'organise autour de la structure naissance-renaissance. « Naître », « renaître » occupent dans le texte de Rolland une position déterminante. Le premier volume du roman, L'Aube, s'ouvre sur une naissance; le dernier volume se clôt sur cette phrase: «Je suis le jour qui va naître »; et l' "Adieu à Jean Christophe » se termine en ces termes: «Mourons, Christophe, pour renaître ».

Dans cette symphonie héroïque, il y a un peu de tout: de la critique musicale, des impressions vives de la vie musicale à Paris mais le grand mérite de Romain Rolland c'est l'analyse profonde du mystère de la création musicale. 


\section{Le narrateur a choisi comme} protagoniste un jeune musicien, génial et très bon, venu de l'Allemagne pour vivre dans la ville des Lumières Paris :

«La délicieuse lumière de Paris! C'était la première chose que Christophe avait aimée dans cette ville; elle le pénétrait, doucement, doucement; peu à peu, elle transformait son cœur, sans qu'il s'en aperçût. Elle était pour lui la plus belle des musiques, la seule musique parisienne. $»^{10}$

En fait, Jean-Christophe Krafft issu du sol romain représente une sorte de double de Romain Rolland lui-même, une image de celui qu'il eût désiré être, puissant comme une force de la nature, et de taille à triompher d'un monde qu'il détestait :

«Christophe est un Krafft, et Kraft, en allemand, veut dire force. ${ }^{11}$

Nous assistons dès le $1^{\text {er }}$ volume à la formation musicale de Jean qui est due à son grand-père, Jean Michel, ainsi qu'à son père, Melchior, qui l'oblige à jouer du piano devant le Grand-Duc Léopold. Dès lors, Christophe se rend compte qu'il aime profondément la musique. C'est la musique qui va l'aider à traverser les graves crises de la vie.

En effet, l'homme qui a le plus d'influence sur les idées du héros, c'est son oncle Gottfried. Il lui a appris que la vérité et la sincérité doivent faire partie intégrante de sa musique. C'est pourquoi Jean est convaincu que le rôle de l'artiste est «de créer le soleil, lorsqu'il n'y en a pas. » ${ }^{12}$

Arrivé au $5^{\text {ème }}$ volume intitulé $L a$ Foire sur la place, c'est le tome où le rôle d'un critique musical tient le plus d'importance et où il s'agit de l'Histoire de la Musique de la Belle Époque. Dans ce volume, Jean Christophe quitte sa mère et ses compatriotes pour pratiquer son métier de pianiste à Paris. C'est là où il rencontre ses deux anciens amis, le marchand de draps, Otto Diener et Sylvain Kohn, employé dans une grande maison d'édition.
Comment fait-il pour se trouver une place dans ce monde vaste et cosmopolite. Les deux grandes parties de cette recherche vont peut-être répondre à la question.

Dans la première partie, il s'agit des espaces clos où se déroulent les séances de musique. Dans la deuxième partie, c'est plutôt la critique de cette période.

La Foire sur la place met à nu la réalité de la société parisienne de l'époque. Christophe est introduit dans cette société par Sylvain Kohn qui le pousse à fréquenter des salons et le conduit à un dîner réunissant de jeunes artistes parisiens et un critique musical nommé Théophile Goujart :

«Mais Christophe, qui
voulait se faire une idée
exacte des musiciens
français, s'acharnait à ne rien
passer; et il admirait le
calme guilleret de ce peuple,
qui se mouvait dans la
contradiction, comme un
poisson dans l'eau. ${ }^{3}$

Christophe s'intéressait avant tout aux musiciens, à la critique musicale, à la littérature, puis au théâtre, mais guère à la peinture :

«Il n'était pas, jusque-là, très touché par la peinture. Il était trop absorbé par l'univers intérieur pour bien saisir le monde des couleurs et des formes. Elles n'agissaient sur lui que par leurs résonnances musicales, qui ne lui en apportaient qu'un écho déformé. ${ }^{14}$

Quand Kohn et Goujart écoutaient Christophe qui jouait au piano, ils affirmaient qu'il avait « la main gauche de Rubinstein $^{15}$ et la main droite de Paderewski $^{16}$. ${ }^{17}$

Christophe pensait que les musiciens doivent de temps en temps pratiquer une lecture approfondie des beaux livres car « la musique ne suffit pas à un musicien : ce n'est pas ainsi qu'il arrivera à dominer le siècle et à s'élever au-dessus du néant. ${ }^{18}$ 
Alors, il commençait par les journaux quotidiens pour connaître la littérature française et l'intérêt accordé à la musique dans les différentes revues de l'époque. Sans citer les titres de ces périodiques, il formule ses opinions propres. Quand Kohn lui montrait « une enquête récente sur l'Art et la Morale », où «l'Amour sanctifiait tout », et «la Sensualité était ferment de l'Art», il ajoutait que «l'Art ne pouvait être immoral», que «la morale était une convention inculquée par une éducation jésuitique », et que seule comptait «l'énormité du Désir. » ${ }^{19}$

Jean Christophe commençait à juger cette société artistique où géographes, historiens, danseuses, peintres et musiciens se mêlaient et se côtoyaient. Peut-on dire que la Belle Epoque est aussi une époque de débauche et de fantaisie? Il constatait aussi que tout le monde écrivait: hommes, femmes et enfants, officiers, comédiens et gens du monde. Une vraie épidémie. ${ }^{20}$

Pour se familiariser avec la langue française et pour pouvoir bien goûter «l'ironie érudite» de quelques critiques artistiques et le «sensualisme cérébral» d'autres critiques non spécialistes de la matière, il était obligé de passer par des expériences et des formations de langue française.

La première impression que notre protagoniste eut des théâtres français était aussi repoussante que celle de ses premières lectures :

«Le théâtre français était pour eux un genre littéraire, donc impur. Comme ils étaient tous littérateurs, ils se défendaient tous de l'être. Toute musique expressive, descriptive, suggestive, en un mot toute musique qui voulait dire quelque chose était taxée d'impure. Dans chaque Français, il y a un Robespierre. Il faut toujours qu'il décapite quelqu'un ou quelque chose, afin de le rendre pur. $\gg^{21}$

Il s'agissait de deux genres de théâtre à Paris, l'un « était à la bonne vieille mode, la façon nationale, la joie de la laideur », le « genre gaulois », et l'autre était «modern-style» qui était «plus raffinée, plus écœurante ", c'était le théâtre des juifs qui mêlaient «les valeurs intellectuels et morales des autres peuples » accompagnés de musique :

«Des auteurs israélites écrivaient pour des actrices israélites des tragédies sur sainte Thérèse. On jouait Chemin de Croix à la Bodinière, L'Enfant Jésus à 1'Ambigu, La Passion à la Porte-Saint-Martin, Jésus à l'Odéon, des Suites d'orchestre sur le Christ, au jardin d'Acclimatation. ${ }^{22}$

Cependant, on se rend compte que Romain Rolland n'a jamais mentionné l'état de la musique de la Belle Époque ni ses musiciens. Le $X X^{\text {ème }}$ siècle s'ouvre par Schönberg $^{23}$ et ses deux disciples Alban Berg et Anton Webern. Pourtant les phares de la création musicale européenne détenait la France avec Ravel ${ }^{24}$ et Debussy. Louis Durey $^{25}$ fut un des premiers à s'inspirer dès 1914 dans son cycle de mélodies l'Offrande Lyrique sur les poèmes de Tagore ${ }^{26}$ traduit par André Gide. Darius Milhaud ${ }^{27}$ était comme Françis Poulenc ${ }^{28}$ lié d'amitié avec le maître autrichien dont il devait diriger la première parisienne de Pierrot Lunaire aux concerts Wiener ${ }^{29}$ en 1921. Schönberg ne fut connu en France qu'après la Deuxième Guerre Mondiale, la France préférant la musique sportive et musclée de Prokofiev ${ }^{30}$, les fantaisies d'Erik Satie ${ }^{31}$ dont Parade avait été représenté en mai 1917 au Châtelet. C'est dire combien ce ballet dont l'argument était de Cocteau, les décors et les costumes de Picasso fait date après les black-out des trois premières années de la guerre. 
Les « Nouveaux Jeunes »: Georges Auric $^{32}$, Louis Durey et Arthur Honegger ${ }^{33}$ donnent un concert dans l'atelier d'un peintre du quartier Montparnasse en hommage à Satie. Ayant pour héraut esthétique Cocteau, ils seront baptisés le lendemain du 16 janvier 1920 comme le Groupe des Six par le critique de Comoedia $^{34}$ : Henri Collet ${ }^{35}$.

D'abord, Christophe allait assister avec Kohn à un concert symphonique dont l'entrée en était commune avec «un music-hall », puis, quand il visitait le théâtre tragique, il constatait que tous les mouvements et même les cris sont prévus comme si la pièce suivait un schéma dramatique :

«Dès les premiers mots, Christophe ne sut plus dans quel monde cela se passait. Les voix des acteurs étaient démesurément amples, lentes, graves, compassés ; elles articulaient toutes les syllabes, comme si elles voulaient donner des leçons de diction; elles paraissaient scander perpétuellement des alexandrins, avec des hoquets tragiques. Les gestes étaient solennels et presque hiérarchiques. » ${ }^{36}$ Quelle éloquence !

Kohn le menait également au théâtre poétique. «Le théâtre est à la poésie ce qu'est l'opéra à la musique. ${ }^{37}$ Mais dans ce genre théâtral, il y avait des rimeurs et non pas des poètes. Il y avait des héros partagés entre la passion et le devoir mais qui «noblement» choisissaient le devoir pour contenter le public.

Il existait aussi des théâtres où $1^{\prime}$ " " on y voyait des meurtres, des viols, des folies, des tortures, yeux arrachés, ventres étripés, tout ce qui pouvait secouer les nerfs et satisfaire la barbarie cachée d'une élite trop civilisée $»^{38}$. A un certain moment, il en avait assez de courir d'un concert à l'autre avec des miettes de symphonie et «des bribes de concerto ».

Le dernier genre théâtral visité par Christophe était l'Opéra-comique où Kohn et Goujart le menèrent pour entendre Pelléas et Mélisande. Mais son avis envers le théâtre français restait le même :

«Rien du tout, continuait Christophe. Pas de musique. Pas de développement. Cela ne se suit pas. Cela ne se tient pas. Des harmonies très fines. De petits effets d'orchestre très bons, de très bon goût. Mais ce n'est rien, rien du tout... $\gg^{39}$

Ensuite, il prit l'habitude de fréquenter les salles de concert pour valoriser la musique parisienne, il remarqua que pendant les concerts il s'agit de prendre pour voisin quelque bon musicien, si possible un compositeur, et de lui faire dire ce qu'il pensait des œuvres qu'on jouait. C'est ainsi que « l'oison pouvait voler » ${ }^{40}$.

Goujart, qui «ne s'était jamais occupé de musique », voulait affirmer que la critique musicale à Paris n'est qu'un métier presque automatique et régulier qui se répète. Selon lui, il s'agit de plaire, «le succès est la loi ; et quand le succès dure, il n'y a qu'à s'incliner » ${ }^{41}$, il «n'attachait pas une importance ridicule à ses opinions; toujours aux ordres de la direction, et prêt à en faire passer les éreintements et les réclames. » $\mathrm{C}$ 'est pourquoi Christophe disait que l'art français ne manque pas de talent mais il manque de caractère. Il protestait contre toute musique artificielle et exigeait l'originalité et le classique avec le nouveau. So rôle plutôt actif que passif, il observait, il comprenait, il agissait.

La suprématie de la musique française étonnait un peu le héros Allemand car il en voyait peu de traces dans le passé : rien de leurs anciens musiciens, rien des grands artistes italiens et rien des grands hommes allemands du XVII ${ }^{\text {ème }}$ et du $X_{\text {XVIII }}{ }^{\text {èe }}$ siècles. Ce qui était aussi étonnant c'est que la musique de cette époque n'avait 
rien avec «la musique française contemporaine $»^{42}$. Dans tous les concerts, il ne voyait que les mêmes artistes et n'entendait que les mêmes morceaux de musique :

\begin{tabular}{llr} 
«Presque rien avant & \multicolumn{1}{c}{ Beethoven. Presque rien } \\
après Wagner. » &
\end{tabular}

Cette musique française ne changeait pas, elle est toujours «douce, pâle, engourdie, anémique, étiolée » mais seuls les titres changent : il était parfois «question de printemps, de midi, d'amour, de joie de vivre, de course à travers les champs », parfois aussi ils «transposaient pour orchestre ou pour piano les tableaux du Louvre, ou les fresques de l'Opéra; ils mettaient en musique Cuyp ${ }^{44}$, Baudry ${ }^{45}$ et Paul Potter ${ }^{46} »$. Nous pouvions aussi trouver des philosophes qui «traitaient en musique des problèmes métaphysiques » ou qui exposaient à travers la musique «un symbole ou d'une religion. »

En fait Jean Christophe avait admiré le temple de la rue Saint-Jacques qui, à son avis, sauva les âmes et la musique de cette période. Dans cet endroit, des élèves actifs «voulaient racheter par leurs pieuses fatigues leurs grands-pères : les Auber ${ }^{47}$, les Adam, et cet archidamné, cet âne diabolique, Berlioz, le diable en personne, diabolus in musica $»^{48}$. Dans dix ans, ils ont transformé la musique française, ils ont connu Bach et ils ont combattu «l'esprit casanier des Français ». Mais la musique de ces gens-là «manque d'air: musique de chambre close, de chaise longue, musique qui ne marche pas » contrairement à Beethoven qui composait sa musique «à travers les champs », « sous le soleil et la pluie, et effrayant les troupeaux par ses gestes et par ses cris ! ${ }^{49}$. Dans ce milieu, la musique prenait un caractère doctrinal et «les concerts devenaient des leçons d'histoire, ou des exemples d'édification ».

D'ailleurs, il fallait bien continuer cette vie, s'il voulait que son art fût connu par la société parisienne. Pour vivre, Christophe a réussi à trouver un poste de professeur de piano et c'est alors qu'avec ses deux élèves, Colette Stevens et sa cousine Germaine Grazia Buontempi, qu'il exprimera sa critique envers la Belle Époque musicale :

«Ils trouvaient des fautes dans Beethoven, donnaient de la férule à Wagner. Pour Berlioz et pour Gluck, ils en faisaient des gorges chaudes. Rien n'existait pour eux, à cette heure de la mode, que Jean-Sébastien Bach et Claude Debussy. ${ }^{50}$

Selon Jean Christophe, la musique «n'était pas faite pour être entendue en fiacre, et qu'elle voulait du recueillement $»^{51}$, elle est une langue naturelle de l'âme ainsi qu'elle «est l'air qu'il respire, le ciel qui l'enveloppe $»^{52}$. Or, ce qu'il rencontre chez les Français et dans la réalité c'est que «la musique semble n'être que l'art de combiner des sons ${ }^{53}$, ils « ne cherchaient dans l'art qu'un instrument de plaisir $»^{54}$ et ils regardaient l'art « comme une sorte de débauche permise ${ }^{55}$.

En effet, Jean Christophe avait une préférence pour la musique classique de Beethoven contrairement aux Français de cette époque qui ne le trouvaient pas distingué et ils le traitaient «de vieux sourd » à cause de sa voix rude. Mais notre héros Allemand voyait que :

«Si Beethoven était venu à Paris, en ce moment, il eût été le lion du jour: c'était fâcheux pour lui qu'il fût mort depuis un siècle. » ${ }^{56}$

Tout au long du volume, la musique de Christophe mûrit et tout en étant attiré par la forme dramatique, il crée le tableau symphonique de David qui était une des figures de la Bible "pour qui il avait une tendresse » :

«Il voyait son David comme un pâtre poétique, au cœur vierge, où dormait l'héroïsme de race plus affinée, plus 
harmonieux de corps et de pensée. » ${ }^{57}$

Ce fameux concert du David prouvait l'originalité artistique de Christophe mais à la suite de ses mauvaises critiques, les musiciens de l'orchestre massacrent la symphonie et la font échouer.

D'ailleurs, en lisant l'histoire de Jeanne d'Arc de Michelet, lors de son rétablissement, Christophe, qui était devenu «d'une sensibilité ridicule » grâce à la bonneté d'une simple domestique, Sidonie; se rend compte qu'il n'était pas bon envers les Français et qu'il les critiquait d'une façon sévère :

«J'ai péché. Je n'ai pas été bon. J'ai manqué de bienveillance. J'ai été trop sévère. Pardon. Ne croyez pas que je sois votre ennemi, vous que je combats! Je voudrais vous faire du bien, à vous aussi... $\gg^{58}$

Sera-t-il imité ? Pourra-t-il convaincre les autres musiciens par son talent? Lors d'une invitation de Mme Roussin à une soirée musicale acceptée à contrecœur, Jean trouvait enfin un admirateur, Olivier Jeannin, qui venait à la soirée seulement pour lui, qui le défendait toujours et qui admirait sa musique :

«C'est un petit poète, qui écrit gentiment! Un de vos admirateurs. Il est bon musicien, et joue bien du piano. [...] il est amoureux de vous. ${ }^{59}$

Il n'est pas besoin de chercher ailleurs que dans cet état d'esprit l'origine et les sources de Jean Christophe, œuvre d'ailleurs sans précédent et sans modèle. Tel était l'avis d'André Gide lorsqu'il notait dans son journal :

« Il m'apparait parfois que ce livre barbare, mal équarri, sans art, sans grâce et de qualités en apparence si peu françaises, reste ce qui a été produit en France de plus important, ou du moins de plus typique par notre génération. " ${ }^{60}$

La supériorité de ce roman sur les autres romans-fleuves du XXème siècle vaut à son auteur le Prix Nobel de littérature en 1915 :

«En hommage rendu au grand idéalisme de ses écrits ainsi qu'à la sympathie, à la vérité avec lesquelles il a peint différents types humains. » ${ }^{61}$

L'art de Romain Rolland témoigne d'un génie fougueux qui contraste singulièrement avec l'esthétisme prudent qui était de règle à la fin du XIXème siècle parce que son effort s'appliquait à provoquer un renouvellement profond des notions sociales et morales. À cet égard, Jean Christophe est une «somme» des idées de l'auteur et nous y voyons l'annonce de tous les combats qu'il dut rencontrer plus tard.

Encouragé par le succès de Jean Christophe, Romain Rolland poursuit des études sur les grands musiciens qui doivent populariser le genre de la musicographie et il entreprend un second grand cycle romanesque, L'Âme enchantée, qu'il achève en 1933 et il meurt en 1944.

«Leur art était un art sans peuple, une musique qui ne s'alimentait que dans la musique, dans le métier. Or Christophe avait l'impression, vraie ou fausse, qu'aucune musique, plus que celle de France, n'aurait eu besoin de chercher un appui en dehors d'elle. Cette plante souple et grimpante ne pouvait se passer d'étai : elle ne pouvait se passer de littérature. ${ }^{62}$ 


\section{Bibliographie}

\section{Corpus:}

ROLLAND (Romain): Jean Christophe, Volume V, La Foire sur la place, Editions de La Bibliothèque électronique du Québec, Collection Classiques du $\mathrm{XX}^{\mathrm{ème}}$ siècle, Volume 58, $384 \mathrm{p}$.

Ouvrages consacrés à Romain Rolland :

BARRẼRE (Jean-Bertrand): Romain Rolland par lui-même, Editions Du Seuil, Paris, 1955, 191p.

CURNIER (Pierre) : Romain Rolland, Jean Christophe, Extraits I, Editions Larousse, Paris, 1953, 83 p.

CURNIER (Pierre) : Romain Rolland, Jean Christophe, Extraits II, Editions Larousse, Paris, 1953, 83 p.

ROBICHEZ (Jacques): Romain Rolland, Pages Choisies, Editions Librairie Hachette, Paris, 1955, 95 p.

ROLLAND (Romain) : Le Voyage intérieur : (songe d'une vie), Editions Albin Michel, Paris, 1959, $396 \mathrm{p}$.

\section{Ouvrages généraux :}

DUCHET (Claude): Manuel d'Histoire Littéraire de la France, Tome V, 1848-1917, Editions Sociales, Paris, 1977.

MARIX-SPIRE (Thérèse) : Les romantiques et la musique: le cas George Sand, 18041838, Volume 1, Nouvelles Editions Latines, 1954, $710 \mathrm{p}$.

PAUTROT (Jean-Louis): La musique oubliée : La nausée, L'écume des jours, A la recherche du temps perdu, Moderato cantabile, Editions Librairie Droz, 1994, Paris, $243 \mathrm{p}$.

VANNIER (Gilles) : Paul Verlaine ou l'enfance de l'art, Champ poétique, Editions Champ Vallon, 1993, Paris, 162 p.

\section{Sitographie :}

Les prix Nobel de littérature: article consulté (en ligne) le 29/3/2015 sur le site : http://www.culturelibre.org/wiki/Les_prix_N obel_de_litt\%C3\%A9rature
Notes

1. VANNIER (Gilles) : Paul Verlaine ou l'enfance de l'art, Champ poétique, Editions Champ Vallon, 1993, Paris, p. 125

2. PAUTROT (Jean-Louis) : La musique oubliée : La nausée, L'écume des jours, A la recherche du temps perdu, Moderato cantabile, Editions Librairie Droz, 1994, Paris, p.133

3. MARIX-SPIRE (Thérèse) : Les romantiques et la musique: le cas George Sand, 1804-1838, Volume 1, Nouvelles Editions Latines, 1954, p.18

4. ROLLAND (Romain): Jean Christophe, Volume V, La Foire sur la place, Editions de La Bibliothèque électronique du Québec, Collection Classiques du XX ${ }^{\text {ème }}$ siècle, Volume 58 , P.192

5. BARRÈRE (Jean-Bertrand): Romain Rolland par lui-même, Editions $\mathrm{Du}$ Seuil, Paris, 1955, p.21

6. LULLY (Jean-Baptiste) est un compositeur et violoniste, français d'origine italienne, de la période baroque.

7. SCARLATTI (Domenico) est un compositeur italien et baroque.

8. ROLLAND (Romain) : Le Voyage intérieur : (songe d'une vie), Editions Albin Michel, Paris, 1959, p. 133

9. CURNIER (Pierre): Romain Rolland, Jean Christophe, Extraits I, Editions Larousse, Paris, p.13

10. ROLLAND (Romain): Jean Christophe, Volume V, La Foire sur la place, Op, Cit, P. 367

11. CURNIER (Pierre): Romain Rolland, Jean Christophe, Extraits I, Op, cit, p.16

12. ROLLAND (Romain): Jean Christophe, Volume V, La Foire sur la place, Op, Cit, P.99

13. Ibid, P.89

14. Ibid, P.333

15. RUBEINSTEIN (Arthur) : (1887-1982) est un pianiste polonais naturalisé 
américain et fut un des interprètes majeurs du XX ${ }^{\text {ème }}$ siècle.

16. PADERESWSKI (Ignacy): (1860-1941) est un pianiste, compositeur, homme politique et diplomate polonais.

17. ROLLAND (Romain): Jean Christophe, Volume V, La Foire sur la place, Op, Cit, P. 78

18. Ibid, P.127

19. Ibid, P.129

20. Ibid, P.136

21. Ibid, p. 96-97

22. Ibid, p.152

23. SCHÖNBERG (Arnold) (1874- 1951) est un compositeur, peintre et théoricien autrichien. Il a posé les fondements de la musique tonale.

24. RAVEL (Maurice) (1875-1937) est un compositeur français. Avec son aîné Claude Debussy, il fut la figure la plus influente de la musique française de son époque.

25. DUREY (Louis) (1888-1979) est un compositeur français.

26. TAGORE (Rabîndranâth) (1861-1941) est un écrivain et philosophe indien, lauréat du Prix Nobel de littérature en 1913.

27. MILHAUD (Darius) (1892-1974) est un compositeur français de musique classique.

28. POULENC (Françis) (1899-1963) est un compositeur et pianiste français.

29. Dans les années 1920-1925, Jean Wiener était un organisateur de concerts.

30. PROKOFIEV (Sergueï) (1891- 1953) est un compositeur russe de musique classique, un pianiste et un chef d'orchestre.

31. SATIE (Erik) (1866-1925) est un compositeur et pianiste français.

32. AURIC (Georges) (1899-1983) est un compositeur français.

33. HONEGGER (Arthur) (1892-1955) est un compositeur suisse, parisien d'adoption.
34. Comoedia est un journal français de presse écrite.

35. COLLET (Henri) (1885-1951) est un compositeur et critique musical français.

36. stophe, Volume $V$, La Foire sur la place, Op, Cit, P.144

37. Ibid, P.147

38. Ibid, P.158

39. Ibid, p.116

40. Ibid, p. 76

41. Ibid, P.169

42. Ibid, P. 87.88

43. Ibid.

44. CUYP (Albert) (1620-1691) est un peintre de paysage néerlandais.

45. BAUDRY (Paul) (1828-1886) est un peintre français.

46. POTTER (Paulus) (1625-1654) est un peintre animalier néerlandais.

47. Auber est un nom de famille d'origine normande.

48. Ibid, p.111-112

49. Ibid

50. Ibid, P.91

51. Ibid, P.86

52. Ibid, P. 124-125

53. Ibid, P.125

54. Ibid, P.162

55. Ibid, P. 194

56. Ibid, P. 182-183

57. Ibid, P. 277

58. Ibid, P.372

59. Ibid, P.379-380

60. CURNIER (Pierre): Romain Rolland, Jean Christophe, Extraits I, Op, Cit, p.14

61. Les prix Nobel de littérature : article consulté (en ligne) le 29/3/2015 sur le site :

http://www.culture-

libre.org/wiki/Les_prix_Nobel_de_litt\% C3\%A9rature

62. ROLLAND (Romain): Jean Christophe, Volume V, La Foire sur la place, Op, Cit, p.122 
